\title{
RESEARCH HIGHLIGHT OPEN IL4I1-driven AHR signature: a new avenue for cancer therapy
}

\author{
Zuli Wang ${ }^{1,2}$, Tiansheng $\mathrm{Li}^{1,2}$, Chao Mao iD ${ }^{1,2}$, Wenliang $\mathrm{Liu}^{3}$ and Yongguang Tao (D) \\ Signal Transduction and Targeted Therapy (2021)6:118 \\ ; https://doi.org/10.1038/s41392-021-00529-z
}

Aryl hydrocarbon receptor (AHR) was considered to be an important pan-tumor therapeutic target, but small molecule inhibitors targeting AHR target gene IDO1 have failed in clinical trials. The recent paper published in Cell by Opitz et al. explained the failure of previous clinical trials and identified new therapeutic targets ${ }^{1}$ (Fig. 1).

AHR is a ligand-activated transcription factor belonging to the basic helix-loop-helix-PER-ARNT-SIM (bHLH-PAS) subgroup of the bHLH superfamily of transcription factors. ${ }^{2}$ Among the ligands of AHR, endogenous tryptophan (Trp) is the most important one. The kynurenine (Kyn) pathway activated by indoleamine-2,3-dioxygenase 1/2 (IDO1/2) or tryptophan-2,3-dioxgenase (TDO2) is considered to be the main tryptophan metabolic pathway in humans, which can produce the AHR agonist Kyn and kynurenic acid (KynA). Interestingly, due to the activation of AHR, tumor cells express high levels of IDO1 and TDO2 to promote plasticity. Since the Kyn-AHR axis inhibits the functions of T cells, the inhibition of Trp-catabolic enzymes also restricts the occurrence and development of tumor. However, the first phase III clinical trial of small molecule inhibitors of IDO1 failed, suggesting that there were other resistance mechanisms against IDO1 inhibition. Interestingly, immune checkpoint blockade (ICB) induces IDO1 and L-amino acid oxidase IL4I1, and IDO1 inhibitors do not block IL $4 \mid 1,{ }^{1}$ indicating that inhibition of novel AHR-mediator IL4I1 is an approach to cancer treatment.

AHR targets change with cell, tissues, and ligands for the context specificity which hinders pan-cancer analysis of AHR activity. To overcome this difficulty, Opitz et al. combined gene expression datasets with natural language processing (NLP) yielding 166 AHR signature genes regulated by AHR across various cell types, diverse ligands, and different AHR agonists or inhibitors.

To assess whether IDO1 and TDO2 were indeed associated with AHR activity, ${ }^{3}$ Opitz et al. performed the high or low group of IDO1 or TDO2 expression and weighted gene co-expression network analysis (WGCNA) across the 32 TCGA tumor entities and found that there was a positive correlation between IDO1 or TDO2 and AHR signaling in 23 tumor types. IDO1 and TDO2 did not appear in the AHRassociated modules, but the L-amino acid oxidase IL4I1 exhibited the highest incidence instead of Trp-catabolic enzymes (TCEs). Interestingly, the classic function of IL4I1 is recognizing and catalyzing the oxidative deamination of phenylalanine (Phe) into phenylpyruvic acid (PP) accompanied by the generation of $\mathrm{H}_{2} \mathrm{O}_{2}$ and $\mathrm{NH}_{3}$, indicating that IL4I1 was a novel potential target gene of AHR. Next, they treated glioblastoma (GBM) cells with IL4I1-derived metabolites, which increased the nuclear localization of AHR and enhanced AHR target genes transcription. Moreover, supernatants of cells with IL4I1 expression suppressed T-cell proliferation. Importantly, high IL4I1 levels were associated with reduced survival in GBM patients.

To explore IL4I1-derived metabolites activate AHR, AHR-proficient cells were exposed to three metabolites including PP, hydroxyphenylpyruvic acid (HPP), and indole-3-pyruvic acid (I3P) that were converted from Phe, tyrosine (Tyr), and Trp by IL4I1. ${ }^{4}$ Surprisingly, the results showed that I3P significantly enhanced the expression of AHR target genes while PP and HPP did not influence their levels in multiple cell types. I3P did not only induce AHR nuclear translocation and transcription, but enhanced the motility of GBM cell and reduced $\mathrm{CD}^{+}{ }^{+} \mathrm{T}$ cells proliferation in an AHR-dependent manner. Compared to the established AHR agonists Kyn and KynA, I3P induced AHR activity at lower concentrations, which signified that I3P represents a novel onco-metabolite. Moreover, the increase of KynA in the cell supernatant came from the metabolism of I3P. Among I3P-derived metabolites, indole-3-acetic acid (IAA) and indole-3-lactic acid (ILA), except indole-3-aldehyde (I3A), could not activate AHR, indicating that IL4I1-converted I3P produced KynA and I3A metabolites leading to AHR activation.

In the clinical significance of IL4I1, the authors found that IL4I1 expression and AHR activity were enhanced in primary cancer tissues and were higher in metastatic melanoma compared to primary melanoma. In fact, although IL4I1 drives the motility of tumor cells, immune modulation is the most enriched function of IL4I1. An accumulation of myeloid-derived suppressor cells (MDSCs) and $T_{\text {regs }}$ a feature of chronic lymphocytic leukemia (CLL), which was strongly associated with high IL4I1 levels. Besides, IL4I1 expression had a positive correlation with AHR activity in patients with CLL. The authors, therefore, utilized aggressive CLL mouse model ${ }^{5}$ to test antitumor immunity effects of IL4I1. Compared to tumor-free mice, it turned out to be that IL4i1 was among the most upregulated genes in tumor-supportive monocytes. Conversely, $I L 4 i 1^{-1-}$ chimeras mice reduced tumor burden, indicating the role of IL4I1 in immune escape. Subsequently, lack of IL $4 I 1$ mitigated $C D 8^{+}$effector T cells $\left(T_{\text {eff }}\right)$ exhaustion through clonally expanded $T_{\text {eff }}$ in TCL1 AT mice. Then, transcriptome analysis of sorted $T_{\text {eff }}$ from tumor-bearing $\mathrm{WT}$ and $I L 4 i 1^{-1-}$ mice verified that IL4I1 deletion increased expression of $\mathrm{T}_{\text {eff }}$ genes and reduced Ahr levels, suggesting that lack of IL4I1 enhances $\mathrm{CD}^{+}{ }^{+}$T-cell function. Last but not least, IL4I1 could develop resistance against ICB due to its immunosuppressive effects. The data showed that anti-PD1 monoclonal antibody nivolumab increased IL4I1 and IDO1 expression and resulted in AHR activation. In combination with anti-PD1 ICB, multiple IDO1 inhibitors that did not limit IL4I1 enzymatic activity has failed on therapy in phase III clinical trial. Together, IL4I1 developed a novel metabolic resistance mechanism against ICB and/or IDO1 inhibitors.

\footnotetext{
${ }^{1} \mathrm{NHC}$ Key Laboratory of Carcinogenesis and Hunan Key Laboratory of Cancer Metabolism, Hunan Cancer Hospital and The Affiliated Cancer Hospital of Xiangya School of

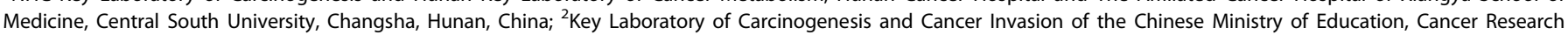

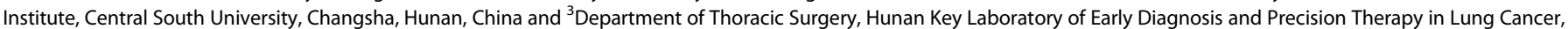
Second Xiangya Hospital, Central South University, Changsha, Hunan, China

Correspondence: Wenliang Liu (liuwenliang@csu.edu.cn) or Yongguang Tao (taoyong@csu.edu.cn)
}

Received: 23 November 2020 Revised: 20 January 2021 Accepted: 7 February 2021

Published online: 10 March 2021 


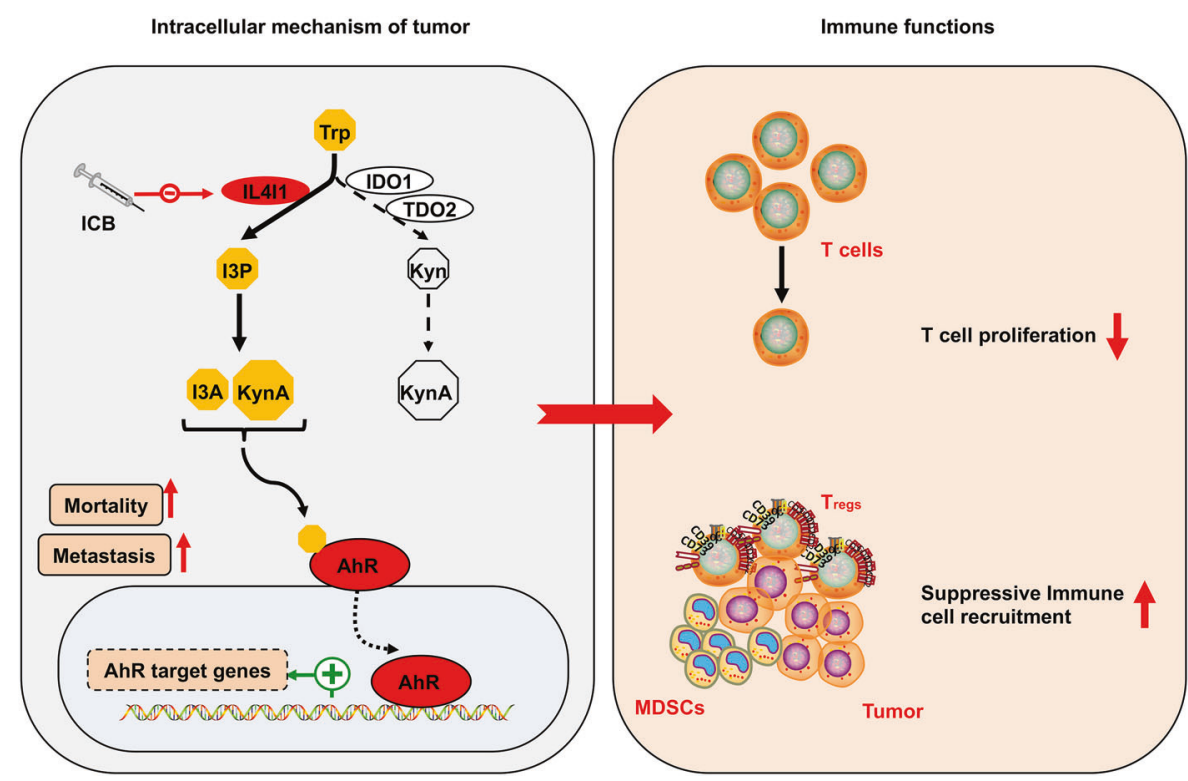

Fig. 1 Newly identified IL4I1 which is induced by immune checkpoint blockade (ICB) mediates AHR signature genes through I3P-KynA/I3A metabolic pathway parallel to IDO1 and/or TDO2-driven AHR signaling. On one hand, IL4I1 can promote cancer cell motility and metastasis, on the other hand, it also inhibits T-cell proliferation and recruits suppressive immune cells

Collectively, combining gene expression analysis and NLP, Opitz et al. first discovered a pan-tissue AHR signature mediator IL4I1 that was linked with cancer cells migration and metastasis in two aspects: (i) IL4I1 does not only play the role in oxidative deamination, but also acts as a tryptophan metabolic enzyme. (ii) The pathway of activation of AHR by the metabolites of IDO1 and TDO2 enzymes is not a suitable immune checkpoint, but the I3A-AHR axis regulated by IL4I1 plays an important role. The new functions of IL4I1 on the recruitment of MDSCs and Tregs in tumor have also been explored. Based on the results that IL4I1 develops a metabolic resistance mechanism against ICB and/or IDO1 by activating $A H R$, this research provided a new and crucial avenue for cancer therapy.

\section{ACKNOWLEDGEMENTS}

This work was supported by the National Natural Science Foundation of China [81672787, 82072594, Y.T.; 81972638, W.L.; 82002916, C.M.], China Postdoctoral Science Foundation [2019M652804, C.M.], Natural Science Foundation of Hunan Province [2020JJ5790, C.M.], Hunan Provincial Key Area R\&D Programs [2019SK2253, Y.T.], and Postdoctoral Foundation of Central South University [220372, C.M.], Shenzhen Science and Technology Program [KQTD20170810160226082], Shenzhen Municipal Government of China [JCYJ20180507184647104].

\section{ADDITIONAL INFORMATION}

Competing interests: The authors declare no competing interests.

\section{REFERENCES}

1. Sadik, A. et al. IL4I1 is a metabolic immune checkpoint that activates the AHR and promotes tumor progression. Cell 182, 1252-1270.e1234 (2020).

2. Murray, I. A., Patterson, A. D. \& Perdew, G. H. Aryl hydrocarbon receptor ligands in cancer: friend and foe. Nat. Rev. Cancer 14, 801-814 (2014).

3. Cervenka, I., Agudelo, L. Z. \& Ruas, J. L. Kynurenines: tryptophanas metabolites in exercise, inflammation, and mental health. Science 357, eaaf9794 (2017).

4. Boulland, M. L. et al. Human IL4I1 is a secreted L-phenylalanine oxidase expressed by mature dendritic cells that inhibits T-lymphocyte proliferation. Blood 110, 220-227 (2007).

5. Hofbauer, J. P. et al. Development of CLL in the TCL1 transgenic mouse model is associated with severe skewing of the T-cell compartment homologous to human CLL. Leukemia 25, 1452-1458 (2011). Attribution 4.0 International License, which permits use, sharing, adaptation, distribution and reproduction in any medium or format, as long as you give appropriate credit to the original author(s) and the source, provide a link to the Creative Commons license, and indicate if changes were made. The images or other third party material in this article are included in the article's Creative Commons license, unless indicated otherwise in a credit line to the material. If material is not included in the article's Creative Commons license and your intended use is not permitted by statutory regulation or exceeds the permitted use, you will need to obtain permission directly from the copyright holder. To view a copy of this license, visit http://creativecommons. org/licenses/by/4.0/.

(c) The Author(s) 2021 